\title{
Modeling a Mobile Group Recommender System for Tourism with Intelligent Agents and Gamification
}

\author{
Patrícia Alves ${ }^{1[0000-0003-3997-311 X]}$, João Carneiro ${ }^{1[0000-0003-1430-5465]}$, Goreti Marreiros ${ }^{1[0000-}$ \\ 0003-4417-8401] and Paulo Novais ${ }^{2[0000-0002-3549-0754]}$ \\ ${ }^{1}$ GECAD - Research Group on Intelligent Engineering and Computing for Advanced Innova- \\ tion and Development, Institute of Engineering, Polytechnic of Porto, 4200-072 Porto, Portugal \\ \{prjaa, jrc, mgt\} @isep.ipp.pt \\ ${ }^{2}$ ALGORITMI Centre, University of Minho, 4800-058 Guimarães, Portugal \\ pjon@di.uminho.pt
}

\begin{abstract}
To provide recommendations to groups of people is a complex task, especially due to the group's heterogeneity and conflicting preferences and personalities. This heterogeneity is even deeper in occasional groups formed for predefined tour packages in tourism. Group Recommender Systems (GRS) are being designed for helping in situations like those. However, many limitations can still be found, either on their time-consuming configurations and excessive intrusiveness to build the tourists' profile, or in their lack of concern for the tourists' interests during the planning and tours, like feeling a greater liberty, diminish the sense of fear/being lost, increase their sense of companionship, and promote the social interaction among them without losing a personalized experience. In this paper, we propose a conceptual model that intends to enhance GRS for tourism by using gamification techniques, intelligent agents modeled with the tourists' context and profile, such as psychological and socio-cultural aspects, and dialogue games between the agents for the post-recommendation process. Some important aspects of a GRS for tourism are also discussed, opening the way for the proposed conceptual model, which we believe will help to solve the identified limitations.
\end{abstract}

Keywords: Group Recommender Systems, Mobile Tourism, ContextAwareness, Gamification, Multi-Agent Systems

\section{$1 \quad$ Introduction}

Since 1992 [1] that Recommender Systems (RS) have been studied to help individual users make better choices $[2,3]$ thus recommending items that intend to better satisfy the users tastes in various domains, each one with its specific challenges, like recommending a movie to watch, a music to listen, a place to visit, a restaurant to lunch, etc. But if to generate accurate individual recommendations is complex, to provide accurate recommendations to groups is even more. The tourism domain has many particularities and is an interesting challenge. To support groups of tourists plan and get accompanied in their excursions can be a very complex task, especially due to the 
group's heterogeneity and conflicting preferences [4]. Millions of tourists participate in planned tours every day, some travel alone, others in groups, but are their needs, interests and curiosity satisfied? Do they enjoy the tours they engaged in? Boratto and Carta [5] state how a group is formed influences its modeling and the predicted recommendations. Groups formed occasionally for a common aim, like travelling together to a specific destination, and that may or may not be acquainted to each other [5] causes this heterogeneity to go deeper. Group Recommender Systems (GRS) are being designed for helping in situations like those, and if they use the capabilities of a mobile device, they can brutally improve the users' experience, bringing new possibilities to explore, like the users' context [6], i.e., the information that surrounds him [7].

In this paper, we introduce a conceptual model that intends to improve the tourists experience in a GRS for tourism by showing concern for their interests, facilitate the post-recommendation process, by proposing the use of an argumentation-based dialogue model between intelligent agents, agents that will accompany the tourists during the tour. Gamification techniques are also proposed to acquire the tourists' profile and motivate them during the tour.

In the next section we present a brief state-of-the-art in GRS for tourism and discuss some current issues. Section 3 introduces dialogue games between intelligent agents and gamification as ways of enhancing the choice process and the tourists' involvement in GRS for tourism, respectively. This section also explains the connection between choice and decision, and how important explanations are in a recommendation. The conceptual model of the GRS for tourism is presented and shortly explained. Section 4 summarizes the contents addressed in the paper and describes what will be done as future work.

\section{Group Recommender Systems for Tourism}

GRS have become an important and challenging theme in the field of RS [8-12] since the group members' preferences can vary, and therefore, to reach a solution that satisfy all the members can be hard to accomplish. It is of extreme importance to guarantee that none of the group members gets too dissatisfied, dissatisfaction that can spread within the group due to the emotional contagion phenomenon [11]. For instance, suppose a travel agency in China that has vacation packages for groups of tourists, with a set of different types of Points of Interest (POI) to visit in a certain country. It is known that Chinese tourists usually travel in groups, either by option or because of impositions [13]. Families, individuals, friends can subscribe a package. But does the package has POI that satisfy all the subscribed members? Although they share the same culture, not all members have the same personality and preferences, but they had no other choice than to choose a predefined package. A vacation that seemed exciting can easily become toilsome. A GRS capable of providing personal and contextual recommendations can be the perfect solution.

Many interesting prototypes of GRS for tourism have and are being proposed to help groups of tourists in the planning of vacations or excursions, usually presenting a 
list of POI to visit. For instance, looking at some of the first GRS for tourism, INTRIGUE (INteractive TouRist Information GUidE) was proposed in 2003 by Ardissono et al. [14] to help (heterogenous) groups of tourists find sightseeing destinations and itineraries in Italy. It is a GRS for mobile and desktop devices where a group member configures the group size, their preferences and characteristics. The group is then divided into subgroups according to those configurations, and recommendations are given to each subgroup grounded by explanations that address potential conflicting requirements.

CATS (Collaborative Advisory Travel System) aims to help a group of friends in planning a ski-holiday $[12,15]$ using a face-to-face collaborative platform (the DiamondTouch interactive tabletop) that uses critiques as a way of giving feedback to recommended POI and iteratively find a final choice.

Garcia et al. [16] developed a GRS for tourist activities, based on the group's tastes, demographic data and places visited in former trips, by extending the $e$ Tourism tool they previously developed for individual tourists. This tool is composed by the Generalist Recommender System Kernel (GRSK), which is a domainindependent taxonomy-driven search engine that manages the group recommendation. It is responsible for aggregating, intersection and incrementally intersection the users' preferences and present a final list of items to recommend.

Travel Decision Forum is a GRS that uses animated characters to represent the group members [17]. The authors state that mutual-awareness and communication are important in order to reach a consensus in the post-recommendation process. For that, the group members configure their preferences incrementally and collaboratively, being able to see the other members' preferences. Since the choice of preferences can be influenced by a person's motivations, the authors implemented a simple way for the members to configure their motivational orientation regarding the other members. This is a very important factor in social interactions that other GRS do not consider, and that we will further discuss later in this paper.

It is perceptible that due to technological limitations at the time, the first GRS were totally dependent of the users' interactions and configurations. Indeed, since the mobile technology was still emerging, the users felt offended for having a "too intelligent" application and argued they could think and decide for themselves, not accepting a too much automatization of the system [18]. However, fifteen years later, the minds "evolved", the users' requirements changed, and many would like to have a more automated system that could think and decide for them, at least regarding recommendations...

In the early 2000's, wireless internet access was very limited and very expensive, but now, that is no longer a problem. The rapid evolution of the wireless internet connections, its throughput, stability, price and massification, also shifted the way (G)RS were being designed and many ideas/approaches found in literature were discontinued. This is a positive reinforcement for creating new and better $(\mathrm{G}) \mathrm{RS}$.

For example, the very recent work by Nguyen and Ricci [4] consists on a chatbased GRS for mobile devices that also allows the group members to become part of the choice process. It is similar to WhatsApp in the way users in a group can exchange messages between them, with the additional features of allowing the users to 
rate previously visited POI and define their mood, so a higher importance is attributed to the user in the preferences aggregation in case he is in a bad mood, tired, etc. The users can classify the recommended POI by liking/disliking them or by classifying one as the best, or comment on them with text and emoticons. This evaluation allows the system to infer users' constraints based on the attributes of the classified POI, and incrementally update the information on a recommended POI with additional explanations, based on those restrictions. Although the system provided higher perceived recommendation quality than the standard benchmark, this approach may not be practical for large and/or occasional groups, since the tested groups were very small, composed of 2 or 3 members. We think it can be very confusing for a group of 20 or more people to chat and exchange opinions in an efficient way. Something else is needed.

\subsection{Important Aspects to Consider in a GRS for Tourism}

To support groups in travel planning is not a simple process and to generate a list of recommendations based on the users' context and preferences is not enough. Other factors need to be considered for a GRS to effectively serve its purposes. For instance, in 2003, Jameson, Baldes and Kleinbauer [17] made the intelligent observation that the recommendation process does not end when a list of recommendations is presented to the user. The users need to decide what to choose from the list, so all the group members get (minimally) satisfied. The authors went even further by stating that it would be short-sighted not to include post-recommendation processes in the design of a $(\mathrm{G}) \mathrm{RS}$, like ways of persuading the other group members to follow a certain recommendation a user finds better. If the process of reaching the final choice has not been delegated to one of the group members, communication and possibly negotiation will be needed between the group members [17]. This falls into the same line of thought that the users need to be somehow involved in the recommendation process, and as mentioned before, a full automatization may not be the perfect solution.

It is evidenced that many people like to know the preferences of other group members, leaning to choose similar preferences [17], either because they would like to please other member(s) or because they tend to avoid conflicts if they previously know what the other users think, like in a real face-to-face scenario. This awareness leads to a sort of collaboration that can help reach a faster consensus. However, this type of behavior is not so linear. Like in a decision-making process, the group members in a choice process can have different intentions, which influence their behaviors and choices. Jameson, Baldes and Kleinbauer [17] address motivation as a way of influencing the choice process. However, motivation is what compels us to fulfil or not our intentions. So, a person's intentions are in the core of a choice, powered by her motivations, and we believe both need to be accounted for. For instance, Phoebe can have an intention to visit a country, but because she cannot go with her boyfriend, she doesn't feel motivated to go, and therefore she won't go unless he does.

As RS can be seen as "tools for helping people to make better choices" [2], how choices are made (the psychology of choice) and how the process of making choices can be supported is of extreme importance [2]. Some GRS are already considering group decision-making (GDM) as an indispensable factor for their success. McCarthy 
et al. [12] developed a face-to-face collaborative GRS for planning skiing vacations. The users reach a consensus by critiquing the items in a list of recommendations during the choice process. Castro, Quesada, Palomares and Martinez [9] proposed a consensus driven GRS, which implements a consensus reaching process used for group decision-making, to iteratively piece together individual recommendations before delivering the group recommendations. The authors concluded that applying a consensus reaching process to group recommendations undoubtedly improved the results and that GRS could benefit from the use of GDM approaches. Marques, Respício and Afonso [19] developed a mobile GRS that uses group collaborative decision-making by using votes. The users model their preferences into the system and give weights to existing restaurants recommendation' platforms. The users have then to democratically elect a restaurant from the generated list of recommended restaurants.

Another extremely important aspect for a RS are the explanations it provides. For instance, Tintarev and Masthoff [20] dedicated a paper to the explanation of recommendations in RS. Explanations can be used with many purposes like: to expose the reasoning behind a recommendation, to gain the users trust and loyalty, to persuade users to buy a recommended item, to increase satisfaction, to help users make better and faster decisions, etc. [20]. The users like to feel the system is not a black box or a computerized oracle that gives advices [21] and that they understand the system. This is even more true when decisions with some impact are involved, like when choosing a honeymoon destination: "Why is the system suggesting I should go to Galápagos in my honeymoon?". Explanations are also very helpful to detect errors in recommendations [20,21], like suggesting Galápagos as a vacation destination because the user visited many websites related to Galápagos since he is researching on Galápagos penguins.

The GRS found in literature are also intrusive in the ways they present the recommendations and are not focused in the tourists' personal interests allied to their context. This causes the tourists to ignore recommendations or ignore the remaining group members. For instance, suppose a group of tourists is visiting a monument with tall towers at some point, and that a member is afraid of heights. The GRS should be capable of warning her that she should not climb those towers because of her fear, avoiding the tourist's discomfort. Or, suppose a tourist is constantly ignoring notifications presented by the GRS in the morning. The GRS should be capable of detecting that the tourist does not like notifications and stop showing them at that time of day.

\section{Intelligent Agents, Dialogue Games and Gamification to Enhance a GRS}

We believe the post-recommendation process can be improved by using intelligent agents and techniques from group decision-making and consensus reaching. So, we propose to solve some of the issues presented before by applying formal dialogue games [22], for agent communication and interaction using argumentation, between intelligent agents modeled to represent the group members. We intend to model each agent with the respective tourist's profile and context, acting on his behalf. So, each 
agent will consider the respective tourist's preferences, personality, socio-cultural aspects, mood, intentions, etc., to choose the POI to visit from the list, engaging in a real time conversation with the other agents by using argumentation. The agents argumentation will also be based on the dynamic argumentation model developed in our previous work [23, 24], and will use dialogues of different types, such as negotiation and deliberation [25], to propose solutions and reach a final consensus on the list of POI to visit that better suits the group's interests and intentions. We believe this strategy can be helpful for large groups, since the agents automatic dialogues will minimize the time the tourists will need to spend in the system to reach a consensus, and will avoid the confusion inherent to chats of large groups of people, simplifying and making the choice process more organized. For example, suppose a group of 30 members where 5 of them are from the same family. The agents from the same family can deliberate together on the POI to visit before dialoguing with the other agents, and then negotiate the POI with the other agents.

The proposed argumentation-based dialogue model will be capable of proposing recommendations and at the same time, due to its self-nature, be capable of explaining the reasons behind those recommendations. We believe this will allow the tourists to feel part of the process and understand it. The dialogue model will also have a high level of expressiveness, meaning the agents will be capable of acting according to different intentions and motivations in the same dialogue, mirroring their tourist, as mentioned in our previous works [26, 27].

Since the tourists will exchange messages in real-time with other tourists in the group, the content of those messages will be studied, content that will influence their agents' dialogues. For that, we will rely on machine-learning techniques such as textmining and natural language processing, in order to study the human dialogues and produce important information in terms of their meaning and the sentiment existent in them.

\subsection{The Conceptual Model}

Fig. 1 shows the architecture for the conceptual mobile GRS. We chose microservices because they allow a better modularity, scalability and the services can be deployed independently, each one with its own database. This means the most suitable programming language(s) can be used for each service, a better faults isolation, continuous delivery and components spread across multiple servers, among others [28]. The communication between the microservices will be asynchronous and through the REST protocol. The API Gateway will be the single-entry point into the system, simplifying the mobile clients' requests and serving as a load balancer for the microservices. The microservices will include the:

\section{Multi-Agent Service.}

This service will be responsible for modeling the intelligent agents according to the tourists' information (profile and context), and other agents necessary to the process, by using the JADE Framework. Here is where the dialogue games between the agents, 
to choose the POI to visit, will also be processed. The agents are also intended to learn the tourists' behavior and context, automatically improving their profile, so better and more proactive recommendations/notifications can be made to the group and/or the individual tourist.

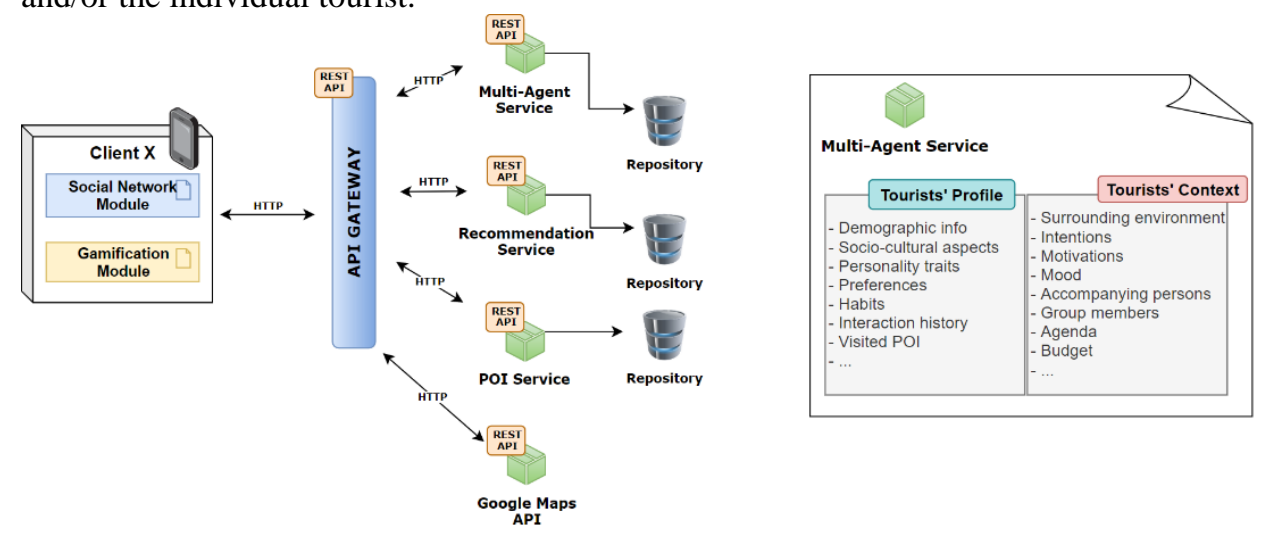

Fig. 1. Left: Architecture of the conceptual mobile Group Recommender System. Right: Information about the tourists, available in the Multi-Agent Service.

Machine-learning classification algorithms will be applied to form (if possible) subgroups of agents/tourists with similar profiles and interests. This can minimize the group's heterogeneity and conflicts of interest, facilitating the consensus reaching in the post-recommendation process and the generation of more precise recommendations to the (sub)groups. This aggregation also intends to promote more socialization and the creation of bounds between the group members. For instance, suppose a group of 50 tourists where 6 of them play Pokémon Go. By comparing the tourists' personality, the agents find out 5 of them have a high openness to experience, agreeableness and low neuroticism. The agents can suggest those members to meet after lunch, at the hotel entrance, to search for Pokémon in the surroundings. Also, suppose the whole group went to visit a monument. If the agents know the personality traits and mood of the tourists, they can suggest a quest for some group members to complete, where they will receive instructions that will make them perform joint tasks to better know the monument, like taking a picture from some important window or collect a certain object that represents the monument's history, promoting their socialization and opening ways of creating bounds between them.

\section{Recommendation Service.}

This service is intended to iteratively run the recommendation algorithm(s) based on the tourists' profile, context and the results obtained from the agents dialogues, presenting the processed recommendations in each iteration, until a final recommendation is accepted by the tourist, in the case of individual recommendations/notifications, or the group/subgroup, in the case of group recommendations/notifications. Since our focus is to work on the post-recommendation process 
that will lead to a consensus on the places to visit and on making more interesting the tourists' experience from the planning to the tour itself, we won't detail on the recommendation algorithm(s).

\section{POI Service.}

This service will be fed by the Google Maps API, or similar, and will be responsible for retrieving all the available POI that match the tour requirements. The list of POI will then be fed into the Recommendation Service so recommendation lists can be generated for the group or subgroups of tourists.

\section{The Social Network Module.}

The proposed GRS will try to embed a Social Network similar to Facebook, composed of secret groups that represent the whole group of tourists for a specific tour, the group of their respective agents, and the subgroups automatically created. The dialogues between the agents will be seen in their respective group, and at the same time, the tourists can post comments in the other available groups. This network is intended to communicate with the Multi-Agent Service, and vice-versa. The list of POI to visit will be presented to the tourists after each agents' dialogue iteration, until they agree in a final itinerary, by posting comments and giving likes/dislikes.

Another important aspect is that our approach is intended to be applied to all group sizes, and not only large ones, because the context in which the group members are is as much important as their profiles. For example, a small group can be formed by 5 hairdressers of different personalities, who do not know each other, but are going to the same congress, i.e., are in the same context, and one of them decides to use the GRS to find other hairdressers to visit the cultural heritage in the congress's country.

\section{The Gamification Module.}

Personalization is a key factor for the success of RS in tourism [29-31]. The more information about the tourist is known better recommendations can be made. Information like the tourists' demographics, personality traits, socio-cultural aspects, habits and preferences can be critical factors for the system's effectiveness. Personality has been evidenced to improve the recommendations made to groups and can even help in the cold-start problem [32-34], since it is demonstrated that personality is strongly related to the users preferences and therefore, correlating the users' personalities and their preferences can help find the preferences of users with similar personalities. For instance, tourists with a high Openness to Experience tend to be more appreciative of the significance of intellectual and artistic pursuits [35] and will probably be more interested in visiting an art exhibit than tourists with low Openness.

To model the tourists' profile will help form groups with similar interests, minimizing the groups' heterogeneity and conflicts of interest. However, the existing GRS are still intrusive and time-consuming in the ways they gather the tourists' profile. The challenge here will be to gather all that information in a non-intrusive and less time-consuming way, and at the same time, motivating and challenging the tourists. 
Gamification can be the leverage we are looking for. It is demonstrated that gamification improves the users' involvement and motivation while learning, working, among other tasks [34, 36-38]. For instance, it has been showed that challenging games motivate students to be more concentrated and committed to the studies, learning significantly better [38]. The use of achievement badges proved to affect the students' behavior motivating them to study [39]. In their work, Mortara et al. [40] present the state-of-the-art of serious games for cultural heritage and state that this approach can be of a tremendous value to learn about the history of a location, its inhabitants and their behaviors. Hence, a GRS for tourism could become more challenging and exciting if we add gaming components to it, like badges for accomplishing certain tasks or mini games to gather the tourists' profile.

Gamification techniques can also be used to personify the agent that represents the tourist in the Multi-Agent Service, transforming it into an Augmented Reality (AR) avatar, visible through the mobile device screen ${ }^{1}$. The avatar would be like the tourist's companion and can play an important role in the system by accompanying the tourist throughout the whole process, helping to decide the itinerary for the group he belongs to, and motivating the tourist during the tour by presenting intelligent information (push-notifications) and proposing personalized challenges according to the tourist's intentions and interests. Why an avatar? It is evidenced that representing the tourist with an avatar can help him feel empathy towards the system [40].

Location-based AR games can have a tremendous potential, and they can be a smarter way of catching the tourists' attention to visit a country's heritage. We propose to transform the whole trip process into a sort of a location-based AR game, where the tourists will have to complete certain personalized "quests" in the POI they visit, using AR features. We hope this will also increase their interest in knowing and learning about a country's heritage, and in a more exciting way.

\section{$4 \quad$ Summary and Future Work}

In this work, we discuss on a novel approach for a Group Recommender System for tourism using agents and gamification. The aim is not to focus on a better algorithm for generating a list of recommendations, but to facilitate the consensus in the postrecommendation process so higher quality and more satisfactory choices can be made, and to enhance the tourists' experience during the whole process, from the planning to the tour itself. We intend to accomplish this by taking advantage of dialogue games using argumentation for the post-recommendation process, between intelligent agents modeled with the tourists' profile and context, and by introducing gaming components in the system that will encourage the tourists' interaction in a more appealing way. The tourists' profile and context will be used to provide more intelligent and personalized recommendations and notifications during the whole tour, to groups of any size. We believe the dialogue games between the agents will be a smarter way of explaining the recommendations to the tourists.

1 Or possibly another device, like Google Glasses ${ }^{\circledR}$, but that is another chapter, not to be addressed in this work. 
Travelling is an emotional experience [41] and therefore, personalization and gamification are becoming a crucial factor for the success of GRS in tourism. In fact, gamification techniques and personalized services will be a major trend for the future of tourism [42]. To motivate the tourists in planning the group tour and configure their profile and context, either implicitly or explicitly, we propose the use of gamification techniques like mini games, badges, trophies, and rankings of the best achievements. An AR avatar is also proposed to represent the tourist's agent and accompany him through the whole process, including during the tour, being responsible for providing personalized and contextual recommendations and push-notifications for the tourist's well-being.

The proposed approach will be thoroughly explained in our future work, and will include, among other tasks, the realization of questionnaires to different cultures in order to develop the model to correlate personality traits with (culture related) touristic preferences, and the development of mini games to implicitly acquire the tourists' personality, preferences and context. The gathered information will be used to model the agents representing the tourists and their avatar. The Social Network prototype will be developed for the post-recommendation choice process and to enable the tourists' online interaction. Intelligent push-notifications, recommendations, other mini games and tasks during the tour will be designed based on the tourists' profile and context. Experiments with real users will be conducted to test the viability of the proposed work and the users' satisfaction.

\section{Acknowledgements}

This work was supported by the GrouPlanner Project (POCI-01-0145-FEDER-29178) and by National Funds through the FCT - Fundação para a Ciência e a Tecnologia (Portuguese Foundation for Science and Technology) within the Projects UID/CEC/00319/2019 and UID/EEA/00760/2019.

\section{References}

1. Goldberg, D., Nichols, D., Oki, B.M., Terry, D.: Using collaborative filtering to weave an information tapestry. Communications of the ACM 35, 61-70 (1992)

2. Jameson, A., Willemsen, M.C., Felfernig, A., de Gemmis, M., Lops, P., Semeraro, G., Chen, L.: Human decision making and recommender systems. Recommender Systems Handbook, pp. 611-648. Springer (2015)

3. Resnick, P., Varian, H.R.: Recommender systems. Communications of the ACM 40, 56-58 (1997)

4. Nguyen, T.N., Ricci, F.: A chat-based group recommender system for tourism. Information Technology \& Tourism 18, 5-28 (2018)

5. Boratto, L., Carta, S.: State-of-the-art in group recommendation and new approaches for automatic identification of groups. Information retrieval and mining in distributed environments, pp. 1-20. Springer (2010) 
6. del Carmen Rodríguez-Hernández, M., Ilarri, S., Hermoso, R., Trillo-Lado, R.: Towards trajectory-based recommendations in museums: evaluation of strategies using mixed synthetic and real data. Procedia Computer Science 113, 234-239 (2017)

7. Lamsfus, C., Wang, D., Alzua-Sorzabal, A., Xiang, Z.: Going mobile: Defining context for on-the-go travelers. Journal of Travel Research 54, 691-701 (2015)

8. Masthoff, J.: Group recommender systems: Combining individual models. Recommender systems handbook, pp. 677-702. Springer (2011)

9. Castro, J., Quesada, F.J., Palomares, I., Martinez, L.: A Consensus-Driven Group Recommender System. International Journal of Intelligent Systems 30, 887-906 (2015)

10. Masthoff, J.: Group recommender systems: aggregation, satisfaction and group attributes. recommender systems handbook, pp. 743-776. Springer (2015)

11. Delic, A., Masthoff, J.: Group Recommender Systems. Proceedings of the 26th Conference on User Modeling, Adaptation and Personalization, pp. 377-378. ACM (2018)

12. McCarthy, K., Salamó, M., Coyle, L., McGinty, L., Smyth, B., Nixon, P.: Group recommender systems: a critiquing based approach. Proceedings of the 11th international conference on Intelligent user interfaces, pp. 267-269. ACM (2006)

13. Nasolomampionona, R.F.: Profile of Chinese outbound tourists: Characteristics and expenditures. American Journal of Tourism Management 3, 17-31 (2014)

14. Ardissono, L., Goy, A., Petrone, G., Segnan, M., Torasso, P.: Intrigue: personalized recommendation of tourist attractions for desktop and hand held devices. Applied artificial intelligence 17, 687-714 (2003)

15. McCarthy, K., McGinty, L., Smyth, B., Salamó, M.: Social interaction in the cats group recommender. Workshop on the social navigation and community based adaptation technologies, (2006)

16. Garcia, I., Sebastia, L., Onaindia, E., Guzman, C.: A group recommender system for tourist activities. International conference on electronic commerce and web technologies, pp. 2637. Springer (2009)

17. Jameson, A., Baldes, S., Kleinbauer, T.: Enhancing mutual awareness in group recommender systems. Proceedings of the IJCAI, (2003)

18. Van Setten, M., Pokraev, S., Koolwaaij, J.: Context-aware recommendations in the mobile tourist application COMPASS. International Conference on Adaptive Hypermedia and Adaptive Web-Based Systems, pp. 235-244. Springer (2004)

19. Marques, G., Respício, A., Afonso, A.P.: A mobile recommendation system supporting group collaborative decision making. Procedia Computer Science 96, 560-567 (2016)

20. Tintarev, N., Masthoff, J.: Explaining recommendations: Design and evaluation. Recommender systems handbook, pp. 353-382. Springer (2015)

21. Herlocker, J.L., Konstan, J.A., Riedl, J.: Explaining collaborative filtering recommendations. Proceedings of the 2000 ACM conference on Computer supported cooperative work, pp. 241-250. ACM (2000)

22. McBurney, P., Parsons, S.: Dialogue games for agent argumentation. Argumentation in artificial intelligence, pp. 261-280. Springer (2009)

23. Carneiro, J., Martinho, D., Marreiros, G., Jimenez, A., Novais, P.: Dynamic argumentation in UbiGDSS. Knowledge and Information Systems 55, 633-669 (2018)

24. Carneiro, J., Alves, P., Marreiros, G., Novais, P.: A Multi-agent System Framework for Dialogue Games in the Group Decision-Making Context. World Conference on Information Systems and Technologies, pp. 437-447. Springer (2019)

25. Walton, D., Krabbe, E.C.: Commitment in dialogue: Basic concepts of interpersonal reasoning. SUNY press (1995) 
26. Carneiro, J., Martinho, D., Marreiros, G., Novais, P.: Arguing with Behavior Influence: A Model for Web-based Group Decision Support Systems. International Journal of Information Technology \& Decision Making 1-37 (2018)

27. Carneiro, J., Saraiva, P., Martinho, D., Marreiros, G., Novais, P.: Representing decisionmakers using styles of behavior: An approach designed for group decision support systems. Cognitive Systems Research 47, 109-132 (2018)

28. Villamizar, M., Garcés, O., Castro, H., Verano, M., Salamanca, L., Casallas, R., Gil, S.: Evaluating the monolithic and the microservice architecture pattern to deploy web applications in the cloud. 2015 10th Computing Colombian Conference (10CCC), pp. 583590. IEEE (2015)

29. Ricci, F.: Travel recommender systems. IEEE Intelligent Systems 17, $55-57$ (2002)

30. Schmidt-Belz, B., Nick, A., Poslad, S., Zipf, A.: Personalized and location-based mobile tourism services. Workshop on "Mobile Tourism Support Systems" in conjunction with Mobile HCI ... (2002)

31. Gavalas, D., Kenteris, M.: A web-based pervasive recommendation system for mobile tourist guides. Personal and Ubiquitous Computing 15, 759-770 (2011)

32. Tkalcic, M., Chen, L.: Personality and recommender systems. Recommender systems handbook, pp. 715-739. Springer (2015)

33. Feil, S., Kretzer, M., Werder, K., Maedche, A.: Using gamification to tackle the cold-start problem in recommender systems. Proceedings of the 19th ACM Conference on Computer Supported Cooperative Work and Social Computing Companion, pp. 253-256. ACM (2016)

34. de CA Ziesemer, A., Müller, L., Silveira, M.S.: Just rate it! Gamification as part of recommendation. International Conference on Human-Computer Interaction, pp. 786-796. Springer (2014)

35. Friedman, H.S., Schustack, M.W.: Personality: Classic theories and modern research. Allyn and Bacon Boston, MA (1999)

36. Hamari, J.: Transforming homo economicus into homo ludens: A field experiment on gamification in a utilitarian peer-to-peer trading service. Electronic commerce research and applications 12, 236-245 (2013)

37. Hamari, J., Koivisto, J., Sarsa, H.: Does gamification work?--a literature review of empirical studies on gamification. 2014 47th Hawaii international conference on system sciences (HICSS), pp. 3025-3034. IEEE (2014)

38. Hamari, J., Shernoff, D.J., Rowe, E., Coller, B., Asbell-Clarke, J., Edwards, T.: Challenging games help students learn: An empirical study on engagement, flow and immersion in gamebased learning. Computers in Human Behavior 54, 170-179 (2016)

39. Hakulinen, L., Auvinen, T., Korhonen, A.: The effect of achievement badges on students' behavior: An empirical study in a university-level computer science course. International Journal of Emerging Technologies in Learning (iJET) 10, 18-29 (2015)

40. Mortara, M., Catalano, C.E., Bellotti, F., Fiucci, G., Houry-Panchetti, M., Petridis, P.: Learning cultural heritage by serious games. Journal of Cultural Heritage 15, 318-325 (2014)

41. Delic, A., Neidhardt, J., Nguyen, N., Ricci, F.: Research Methods for Group Recommender System. CEUR-WS (2016)

42. Xu, F., Tian, F., Buhalis, D., Weber, J., Zhang, H.: Tourists as mobile gamers: Gamification for tourism marketing. Journal of Travel \& Tourism Marketing 33, 1124-1142 (2016) 